CALT-68-2123

June 1997

\title{
Notes on Branes in Matrix Theory
}

\author{
Esko Keski-Vakkuri ${ }^{1}$ and Per Kraus ${ }^{2}$ \\ California Institute of Technology \\ Pasadena CA 91125, USA \\ email: esko or perkraus@theory.caltech.edu
}

\begin{abstract}
:
We study the effective actions of various brane configurations in Matrix theory. Starting from the $0+1$ dimensional quantum mechanics, we replace coordinate matrices by covariant derivatives in the large $\mathrm{N}$ limit, thereby obtaining effective field theories on the brane world volumes. Even for noncompact branes, these effective theories are of Yang-Mills type, with constant background magnetic fields. They correspond to certain limits of Born-Infeld actions. We also consider the effect of compactifying transverse directions. Finally, we analyze a scattering process involving a recently proposed background representing a classically stable D6+D0 brane configuration. We compute the potential between this configuration and a D0-brane, and show that the result agrees with supergravity.
\end{abstract}

${ }^{1}$ Work supported in part by a DOE grant DE-FG03-92-ER40701.

${ }^{2}$ Work supported in part by a DOE grant DE-FG03-92-ER40701 and by a DuBridge Fellowship. 


\section{Introduction}

Matrix theory [1] purports to be a complete, non-perturbative formulation of $\mathrm{M}$ theory. Since a great deal is known about the physics of $M$ theory in various corners of moduli space, much effort has recently been directed towards verifying that such physics can be recovered from Matrix theory. In this paper we will be primarily interested in studying the description of D-branes that emerges when matrix theory is compactified on a circle, yielding the type IIA theory. Some salient features of D-branes in IIA theory which one would like to obtain from Matrix theory include the following: there exist Dp-branes for $\mathrm{p}=0,2,4,6,8$; the low energy dynamics of $\mathrm{k}$ parallel $\mathrm{Dp}$-branes is described by a supersymmetric $\mathrm{p}+1$ dimensional $\mathrm{U}(\mathrm{k})$ gauge theory; the (not necessarily low energy) dynamics of a single D-brane is described by a Born-Infeld type action. In addition, one knows the supergravity fields produced by D-branes and so also the corresponding small angle scattering amplitudes of several such objects.

Banks, Seiberg, and Shenker [2] (and S.-J. Rey, in an unpublished work [3]) took some first steps towards deriving the effective actions of D-branes from Matrix theory. They identified matrix assignments corresponding to D-branes by identifying the appropriate central charges in the 11 dimensional light cone gauge supersymmetry algebra. Then by analyzing the fluctuations around such backgrounds and replacing large $\mathrm{N}$ commutators by Poisson brackets, they were able to recover the general structure of the known effective actions to quadratic order in fields. Here, our goal is similar, but we wish to go beyond quadratic order and to keep careful track of numerical factors. To do so successfully, we found it necessary to replace matrix commutators not by Poisson brackets but rather by the commutators of covariant derivatives, much as one does when considering toroidal compactification. By so doing, we are able to obtain gauge invariant actions beyond quadratic order, and to see certain features of the Born-Infeld action emerging. But as we will discuss, some puzzles remain.

There have been a number of studies comparing D-brane scattering amplitudes obtained from Matrix theory and from supergravity. On the Matrix theory side, the procedure involved computing the effective action of a finite $\mathrm{N}$ matrix configuration, and then extrapolation to large N. Strictly speaking, this procedure is inconsistent as the backgrounds require that certain finite $\mathrm{N}$ matrices have a commutator proportional to the identity, even though such matrices cannot exist. In our approach, we avoid this problem by taking $N \rightarrow \infty$ from the outset. As a specific application, we consider the scattering of a D0-brane from a configuration composed purely of D6 and D0 branes [4], which has not been studied before. We find complete agreement at the one loop level between Matrix theory and supergravity.

The remainder of this paper is organized as follows. In section 2 we rewrite the Matrix 
theory action in a manner suitable for the study of D-branes. Sections 2.1 and 2.2 focus on the effective action for D2-branes, and section 2.3 on the effective action for D4-branes. In section 3 we compute the potential between a D0-brane and a D6+D0 configuration, and in section 4 we discuss some of our results.

\section{Fluctuations of branes in Matrix theory}

The action governing Matrix theory is gotten by dimensionally reducing the action of 10 dimensional Super Yang-Mills theory to $0+1$ dimensions. In string units $\left(2 \pi \alpha^{\prime}=1\right)$ the bosonic part of the Lagrangian is

$$
\mathcal{L}_{B}=\operatorname{Tr} L_{B} ; L_{B}=\frac{1}{2 g}\left\{\left(D_{0} X_{I}\right)^{2}+\frac{1}{2}\left[X_{I}, X_{J}\right]^{2}\right\}
$$

where

$$
D_{0}=\partial_{t}-i\left[A_{0}, \cdot\right]
$$

and $I=1,2, \ldots, 8,9$. A p-brane background is described by

$$
\begin{aligned}
& X_{r}=U_{r} ; r=1,2, \ldots, p \\
& X_{i}=0 ; i=p+1, \ldots, 9
\end{aligned}
$$

where $U_{r}, r=1, \ldots, p$ are certain matrices to be specified later.

Fluctuations around this background are denoted by $A_{r}, \phi_{i}$,

$$
\begin{aligned}
X_{r} & =U_{r}+A_{r} \\
X_{i} & =\phi_{i} .
\end{aligned}
$$

Following Banks, Seiberg and Shenker [2], we substitute the above expansions into the Lagrangian $L_{B}$, and then regroup and rename the terms so that we arrive at the following form:

$$
L_{B}=\frac{1}{2 g}\left\{-\frac{1}{2}\left(F_{\mu \nu}\right)^{2}+\left(D_{\mu} \phi_{i}\right)^{2}+\frac{1}{2}\left[\phi_{i}, \phi_{j}\right]^{2}+i\left[U_{r}, U_{s}\right] F_{r s}+\frac{1}{2}\left[U_{r}, U_{s}\right]^{2}\right\},
$$

where $\mu, \nu=0,1, \ldots, p ; r, s=1, \ldots, p$ and

$$
\begin{aligned}
F_{0 r} & =-F_{r 0}=\partial_{0} A_{r}+i\left[U_{r}, A_{0}\right]-i\left[A_{0}, A_{r}\right] \\
F_{r s} & =-i\left[U_{r}, A_{s}\right]+i\left[U_{s}, A_{r}\right]-i\left[A_{r}, A_{s}\right] \\
D_{0} \phi_{i} & =\partial_{0} \phi_{i}-i\left[A_{0}, \phi_{i}\right] \\
D_{r} \phi_{i} & =-i\left[U_{r}, \phi_{i}\right]-i\left[A_{r}, \phi_{i}\right] .
\end{aligned}
$$


(In the above, $\left(D_{\mu} \phi_{i}\right)^{2}=\left(D_{0} \phi_{i}\right)^{2}-\left(D_{r} \phi_{i}\right)^{2}$ etc.)

So far, we have not done anything other than reorder the terms and give them new names. Now we try to interpret the result in the context of some particular p-brane backgrounds.

\subsection{Infinite membrane backgrounds $(p=2)$}

The 11 dimensional SUSY algebra admits central charges corresponding to the presence of membranes. Banks, Seiberg and Shenker [2] computed the central charges starting from the Matrix theory action and found

$$
Z_{r s}=-\frac{i}{2 \pi} \operatorname{Tr}\left[X_{r}, X_{s}\right]
$$

It follows immediately that $Z_{r s}$ vanishes for finite $N$. On the other hand, it has recently been proposed by Susskind [5] that the finite $N$ version of Matrix theory is to be interpreted as describing a finite longitudinal momentum sector of $M$ theory quantized in light cone gauge. The vanishing of $Z_{r s}$ at finite $N$ can be understood in this light, for a membrane carrying finite momentum must necessarily be compact and so carry no net central charge.

Ref. [1] discussed a formal method for constructing membrane backgrounds in the $N \rightarrow \infty$ limit. The construction uses canonical variables $Q, P$ obeying the commutation relation

$$
[Q, P]=\frac{2 \pi i}{N}
$$

Since this relation cannot be satisfied at finite $N$, we will instead take the $N \rightarrow \infty$ limit in terms of the rescaled variables [6]

$$
U_{1}=Q \sqrt{N z_{12}} ; U_{2}=P \sqrt{N z_{12}} \text {. }
$$

Taking the spectrum of $P, Q$ to go from 0 to $2 \pi$, the area of the membrane is $A=$ $(2 \pi)^{2} N z_{12}$, becoming infinite in the $N \rightarrow \infty$ limit. The commutation relation

$$
\left[U_{1}, U_{2}\right]=2 \pi i z_{12}
$$

can be represented by differential operators acting on a space of functions. More formally, the $N$ dimensional vector space $V_{N}$ on which the matrices $X$ act will be replaced by an infinite dimensional space $V_{\infty}$ of functions on the membrane worldvolume. The membrane

\footnotetext{
${ }^{1}$ with the normalization corresponding to conventions in this paper
} 
background $U_{1}, U_{2}$ is represented by differential operators acting on $V_{\infty}$, and fluctuations will be represented by elements of $V_{\infty}$, i.e., functions on the membrane worldvolume.

A simple representation of $U_{1,2}$ found in the literature consists of taking

$$
U_{1}=2 \pi i z_{12} \partial_{y} \quad ; U_{2}=y
$$

in the $N \rightarrow \infty$ limit. As Aharony and Berkooz [6] have pointed out, a cosmetic flaw of this representation is that a membrane carries two spatial coordinates whereas only one is manifest in the above equation.

There exists, however, a more "natural" representation than the one given above. Membranes carry a charge, which in Matrix theory arises from a U(1) subgroup of the $\mathrm{U}(\mathrm{N})$ symmetry, or in the continuum limit, the $\mathrm{U}(1)$ subgroup of $\mathrm{U}(\infty)$ which is isomorphic with the infinitesimal area preserving diffeomorphisms of the membrane. Specifically, the membrane charge is associated with the flux of a $\mathrm{U}(1)$ magnetic field living on the membrane world volume. Thus, we shall represent $U_{1,2}$ by

$$
\begin{aligned}
& U_{1} \leftrightarrow i \partial_{x_{1}}+a_{1}\left(x_{1}, x_{2}\right) \\
& U_{2} \leftrightarrow i \partial_{x_{2}}+a_{2}\left(x_{1}, x_{2}\right)
\end{aligned}
$$

where $\left(a_{1}, a_{2}\right) \equiv \mathbf{a}$ is the background $\mathrm{U}(1)$ field, and $\left[U_{1}, U_{2}\right]=i(\nabla \times \mathbf{a})$ is the magnetic flux density on the membrane: the 2-brane charge density. A constant membrane charge density, as in (10), is given by

$$
a_{1}=-z_{12} \pi x_{2} \quad, \quad a_{2}=z_{12} \pi x_{1} .
$$

In this representation both of the membrane coordinates and the background magnetic field are manifest. To avoid confusion, let us point out that although expressions similar to (12),(13) appear in the context of toroidal compactification [9], here the motivation and the interpretation are quite different. We have not T-dualized anything, the two coordinates $x_{1}, x_{2}$ parametrize the infinite membrane, $\left(x_{1}, x_{2}\right) \in R^{2}$.

In the $N \rightarrow \infty$ limit, the trace operation is replaced by integration over the spatial membrane worldvolume coordinates $x_{1}, x_{2}$ :

$$
\operatorname{Tr} \leftrightarrow \sigma_{0} \int d x_{1} d x_{2},
$$

where the normalization factor $\sigma_{0}$ is the density of D0-branes on the infinite membrane,

$$
\sigma_{0}=\frac{1}{(2 \pi)^{2} z_{12}} .
$$

$\sigma_{0}$ is introduced in order to preserve the interpretation of the rank of the matrices as the number of D0-branes $N=\operatorname{Tr} \mathbf{1}_{N}$; we represent the unit matrix $\mathbf{1}_{N}$ by 1 . All other $N \times N$ 
matrices are represented by functions on the membrane worldvolume:

$$
\begin{aligned}
A_{0} & \leftrightarrow A_{0}\left(x_{1}, x_{2}\right) \\
A_{r} & \leftrightarrow A_{r}\left(x_{1}, x_{2}\right) \\
\phi_{i} & \leftrightarrow \phi_{i}\left(x_{1}, x_{2}\right)
\end{aligned}
$$

(We have suppressed the dependence on the worldvolume time coordinate.) The matrix commutators which involve $U_{r}$ become commutators of operators and functions, e.g.

$$
\left[U_{r}, A_{0}\right] \leftrightarrow\left[i \partial_{r}+a_{r}\left(x_{1}, x_{2}\right), A_{0}\left(x_{1}, x_{2}\right)\right]
$$

The matrix commutators which do not involve $U_{r}$ become commutators of functions, e.g.

$$
\left[\phi_{i}, \phi_{j}\right] \leftrightarrow\left[\phi_{i}\left(x_{1}, x_{2}\right), \phi_{j}\left(x_{1}, x_{2}\right)\right]
$$

In the case of a single membrane, these will be zero.

Now we can see what happens to $L_{B}$. It becomes a $2+1$ dimensional Lagrangian density with the terms

$$
\begin{array}{rcccr}
F_{0 r} & = & \partial_{0} A_{r}+i\left[U_{r}, A_{0}\right]-i\left[A_{0}, A_{r}\right] & \leftrightarrow & \partial_{0} A_{r}(x)-\partial_{r} A_{0}(x) \\
F_{12}= & -i\left[U_{1}, A_{2}\right]+i\left[U_{2}, A_{1}\right]-i\left[A_{1}, A_{2}\right] & \leftrightarrow & \partial_{1} A_{2}(x)-\partial_{2} A_{1}(x) \\
D_{0} \phi_{i}= & \partial_{0} \phi_{i}-i\left[A_{0}, \phi_{i}\right] & \leftrightarrow & \partial_{0} \phi_{i}(x) \\
D_{r} \phi_{i}= & -i\left[U_{r}, \phi_{i}\right]-i\left[A_{r}, \phi_{i}\right] & \leftrightarrow & \partial_{r} \phi_{i}(x)
\end{array}
$$

So, we get

$$
\mathcal{L}_{B}=\operatorname{Tr} L_{B} \leftrightarrow \sigma_{0} \int d^{2} x \frac{1}{2 g}\left\{-\frac{1}{2}\left(F_{\mu \nu}\right)^{2}+\left(\partial_{\mu} \phi_{i}\right)^{2}-4 \pi z_{12} F_{12}-\left(2 \pi z_{12}\right)^{2}\right\} .
$$

As a check, using the last term we can work out the tension of the membrane to be

$$
T_{2}=\frac{1}{2 \pi} T_{0}
$$

which is correct. Notice that a key feature of our representation in the $N \rightarrow \infty$ limit is the vanishing of commutators such as (18). This is in contrast to the approach in [2], where the authors represented the commutators of matrices by Poisson brackets. Such a representation yields nonvanishing contributions such as

$$
\left\{A_{r}, A_{s}\right\}
$$

to the $F_{r s}$ component of the field strength, which is cumbersome from the D2-brane point of view where one expects to obtain a $U(1)$ symmetry. This puzzle seems to be related to the distinction between infinite and finite membranes; for discussion, see [6]. 
Note also that even if the commutators vanish for a single infinite membrane, we will obtain nonvanishing commutators later when we discuss the case of multiple membrane backgrounds.

It is interesting to examine the relation between the effective action (19) which was derived from Matrix theory, and the analogous Born-Infeld action for a D2-brane in IIA theory. The latter is

$$
L_{B I}=-T_{2} \int d^{2} x \sqrt{-\operatorname{det}\left[\eta_{\mu \nu}+F_{\mu \nu}\right]},
$$

here for simplicity we keep only the $f_{\mu \nu}$ dependent part. One possibility is to expand $L_{B I}$ to quadratic order

$$
L_{B I} \approx T_{2} \int d^{2} x\left\{\frac{1}{2}\left(F_{\mu \nu}\right)^{2}-1\right\} .
$$

It is easy to see that the relative coefficient of the $\left(F_{\mu \nu}\right)^{2}$ and the constant pieces disagrees with (19). The reason for the mismatch is that the membrane has a large momentum in the 11th direction, which corresponds to a worldvolume magnetic field in the IIA description. We should instead substitute $F_{\mu \nu} \rightarrow f_{12}+F_{\mu \nu}$, where $f_{12}$ denotes the magnetic background field and $F_{\mu \nu}$ fluctuations about the background, and expand (21) for large $f_{12}$. In this case we get

$$
L_{B I}^{\left(F_{12}\right)} \approx \int d^{2} x\left\{\frac{T_{2}}{2 f_{12}}\left(F_{\mu \nu}\right)^{2}-T_{2} f_{12}\right\} .
$$

If we set $f_{12}=2 \pi z_{12}$, which is the value of the magnetic field introduced in the Matrix description via (12), (13), then we find

$$
L_{B I}^{\left(f_{12}\right)}=2 L_{B},
$$

and we reproduce (19) up to an overall factor of 2. However, there is a puzzling feature of this correspondence. The magnetic field on the D2-brane should be equal to the density of D0-branes bound to it, which we have already argued to be given by $\sigma_{0}$. But $\sigma_{0}$ and $f_{12}=2 \pi z_{12}$ are not equal, and indeed have inverse dependence on the parameter $z_{12}$. Thus the relation between the Matrix and IIA descriptions remains unclear.

Consider now a background configuration of $k$ parallel infinite membranes in the transverse directions $X_{1}, X_{2}$. At finite $N$, this is represented by block diagonal $k N \times k N$ matrices, $X_{1}$ has $k$ copies of the " $N \times N$ matrix" $Q$ in the diagonal, and $X_{2}$ has $k$ copies of $P$. Correspondingly, for $k$ infinite membranes we take

$$
\begin{aligned}
& U_{1} \leftrightarrow\left(i \partial_{1}+a_{1}\right) \otimes \mathbf{1}_{k} \\
& U_{2} \leftrightarrow\left(i \partial_{2}+a_{2}\right) \otimes \mathbf{1}_{k}
\end{aligned}
$$

where $\mathbf{1}_{k}$ is the unit $k \times k$ matrix. Thus,

$$
\left[U_{1}, U_{2}\right]=2 \pi i z_{12} \mathbf{1}_{k} .
$$


This background breaks the $U(\infty)$ symmetry down to an $U(k)$ symmetry. The fluctuations $A_{r}, \phi_{i}$ are not block diagonal, which would correspond to fluctuations within each brane, but also contain off diagonal components corresponding to strings connecting distinct membranes, thereby accounting for the full $k N \times k N$ matrix structure. The matrices can be represented as a sum of tensor products of $N \times N$ matrices and generators of the $U(k)$ Lie algebra. The $N \times N$ parts, in the $N \rightarrow \infty$ limit, are replaced by functions on the membrane - thus (16) is now replaced by

$$
\begin{aligned}
A_{0} & \leftrightarrow A_{0}^{0}\left(x_{1}, x_{2}\right) \mathbf{1}_{k}+A_{0}^{a}\left(x_{1}, x_{2}\right) T^{a} \\
A_{r} & \leftrightarrow A_{r}^{0}\left(x_{1}, x_{2}\right) \mathbf{1}_{k}+A_{r}^{a}\left(x_{1}, x_{2}\right) T^{a} \\
\phi_{i} & \leftrightarrow \phi_{i}^{0}\left(x_{1}, x_{2}\right) \mathbf{1}_{k}+\phi_{i}^{a}\left(x_{1}, x_{2}\right) T^{a} .
\end{aligned}
$$

where $T^{a}$ are the traceless generators of the $S U(k)$ Lie algebra. Commutators thus become commutators of fields in the adjoint representation of $U(k)$, e.g. (18) becomes

$$
\left[\phi_{i}\left(x_{1}, x_{2}\right), \phi_{j}\left(x_{1}, x_{2}\right)\right]_{k}
$$

and the $S U(k)$ part will survive.

The trace over $k N \times k N$ matrices is now represented as follows

$$
\operatorname{Tr} \leftrightarrow \sigma_{0} \int d x_{1} d x_{2} \operatorname{Tr}_{k},
$$

where $\operatorname{Tr}_{k}$ is the trace over the $k \times k$ structure. Note that the total membrane charge of the multimembrane configuration is

$$
\begin{aligned}
Z_{12}=-\frac{i}{2 \pi} \operatorname{Tr}\left[X_{1}, X_{2}\right] & \leftrightarrow \sigma_{0} \int d x_{1} d x_{2} z_{12} \operatorname{Tr}_{k} \mathbf{1}_{k} \\
& =k \cdot(\text { charge of a single membrane })
\end{aligned}
$$

as it should be. Plugging everything into the Lagrangian (6), we obtain

$$
\mathcal{L}_{B} \leftrightarrow \sigma_{0} \int d^{2} x \frac{1}{2 g} \operatorname{Tr}_{k}\left\{-\frac{1}{2}\left(F_{\mu \nu}\right)^{2}+\left(D_{\mu} \phi_{i}\right)^{2}+\frac{1}{2}\left(\left[\phi_{i}, \phi_{j}\right]_{k}\right)^{2}-4 \pi z_{12} F_{12}-\left(2 \pi z_{12}\right)^{2} \mathbf{1}_{k}\right\}
$$

where

$$
\begin{aligned}
F_{0 r} & \leftrightarrow \partial_{0} A_{r}(x)-\partial_{r} A_{0}(x)-i\left[A_{0}(x), A_{r}(x)\right]_{k} \\
F_{12} & \leftrightarrow \partial_{1} A_{2}(x)-\partial_{2} A_{1}(x)-i\left[A_{1}(x), A_{2}(x)\right]_{k} \\
D_{0} \phi_{i} & \leftrightarrow \partial_{0} \phi_{i}(x)-i\left[A_{0}(x), \phi_{i}(x)\right]_{k} \\
D_{r} \phi_{i} & \leftrightarrow \partial_{r} \phi_{i}(x)-i\left[A_{r}(x), \phi_{i}(x)\right]_{k}
\end{aligned}
$$

We can also check the gauge transformation properties directly. The Matrix Lagrangian (6) is invariant under the $U(k N)$ gauge transformations

$$
\begin{aligned}
\delta A_{r} & =-i\left[U_{r}, \lambda\right]+i\left[\lambda, A_{r}\right] \\
\delta \phi_{i} & =i\left[\lambda, \phi_{i}\right] \\
\delta A_{0} & =\partial_{0} \lambda+i\left[\lambda, A_{0}\right]
\end{aligned}
$$


where the gauge transformation parameter $\lambda$ (and everything else) is a $k N \times k N$ matrix. In the $N \rightarrow \infty$ limit these become

$$
\begin{aligned}
\delta A_{r}(x) & \leftrightarrow \partial_{r} \lambda(x)+i\left[\lambda(x), A_{r}(x)\right]_{k} \\
\delta \phi_{i}(x) & \leftrightarrow i\left[\lambda(x), \phi_{i}(x)\right]_{k} \\
\delta A_{0}(x) & \leftrightarrow \partial_{0} \lambda(x)+i\left[\lambda(x), A_{0}(x)\right]_{k}
\end{aligned}
$$

and give the correct $U(k)$ gauge symmetry of (31).

\subsection{Wrapped membrane backgrounds $(\mathrm{p}=2)$}

We now compactify the Matrix theory on a two dimensional torus $T^{2}$ in the $X_{1}, X_{2}$ directions. This corresponds to compactifying $\mathrm{M}$ theory on $T^{2} \times S^{1}$, since one spatial direction was already compactified. We will then consider backgrounds of transverse membranes which wrap around the torus $T^{2}$. As a result of the compactification, there will be additional degrees of freedom in the theory, due to strings which wind around the compact directions. As explained by Ganor, Ramgoolam and Taylor [9], they can be accomodated in the Matrix theory by replacing each partonic D0-brane by a countably infinite number of copies of it on the noncompact covering space of the torus. The vector space $V$, on which the matrices act, then has a tensor product structure

$$
V=V_{N} \otimes H^{2}
$$

The $N$-dimensional space $V_{N}$ is multiplied by a countably infinite dimensional space $H^{2}$ associated with the countable infinity of copies of D0-branes and strings stretching between them?.

The description of the Matrix model becomes simpler upon performing T-duality in directions $X_{1}, X_{2}$. The torus $T^{2}$ is replaced by its dual torus $\hat{T}^{2}$, and the $0+1$ dimensional quantum mechanics becomes a $2+1$ dimensional $U(N)$ super-Yang-Mills theory [9, 11].

Instead of analyzing the fluctuations of a wrapped transverse membrane in the T-dual $2+1$ dimensional Matrix theory, we will try to recover a Lagrangian for the massless fluctuations directly from the $0+1$ dimensional theory on the initial torus $T^{2}$. Our approach will be to construct a membrane on the covering space and then to demand periodicity of the spatial coordinates. We consider a constant density $\sigma_{0}$ of D0-branes on the covering space. The total number of D0-branes is thus infinite, consistent with a nonvanishing value of the membrane charge, $\operatorname{Tr}\left[X_{1}, X_{2}\right] \neq 0$. The construction then proceeds much as

\footnotetext{
${ }^{2}$ For a complementary investigation on winding supermembranes in eleven dimensions, see [10].
} 
before: we represent the membrane background $U_{1,2}$ as covariant derivatives with a $\mathrm{U}(1)$ magnetic field, acting on functions of the membrane world volume,

$$
\begin{aligned}
& U_{1} \leftrightarrow i \partial_{1}+a_{1} \\
& U_{2} \leftrightarrow i \partial_{2}+a_{2}
\end{aligned}
$$

where $\mathbf{a}=\left(a_{1}, a_{2}\right)=z_{12} \pi\left(x_{2},-x_{1}\right)$ as before. However, now the coordinates $x_{1}, x_{2}$ have a finite range:

$$
0 \leq x_{r} \leq L_{r} \quad, r=1,2
$$

where $L_{1}, L_{2}$ are the sizes of the torus $T^{2}$, and we demand that all quantities are periodic up to gauge transformations. With this range for the coordinates $x_{1}, x_{2}$, the membrane wraps once around the torus. The trace is represented as in (14), leading to a finite value of total charge

$$
Z_{12}=-\frac{i}{2 \pi} \sigma_{0} \int d^{2} x\left[U^{1}, U^{2}\right]=\sigma_{0} z_{12} L_{1} L_{2}=\frac{L_{1} L_{2}}{(2 \pi)^{2}} .
$$

for the wrapped membrane.

The fluctuation analysis proceeds as before, and leads to the same effective $2+1$ dimensional Lagrangian (19) as before. The tension of the membrane can be checked to be given by the relation (20), as it should.

Consider now 2 parallel membranes wrapped on $T^{2}$. In addition to the excitations of open strings within each membrane, there are excitations of strings which interpolate between the two membranes. As discussed in [8], these strings do not have winding modes because they are all homotopic to each other. Effectively, these strings behave as if the spacetime would be non-periodic. However, the homotopy property and the periodicity of the torus is seen in the possibility of introducing Wilson lines which correspond to non-trivial gauge holonomy. We will return to this issue shortly.

For two parallel membranes, we could write

$$
\begin{aligned}
& U_{1} \leftrightarrow\left(i \partial_{1}+a_{1}\right) \otimes \mathbf{1}_{2} \\
& U_{2} \leftrightarrow\left(i \partial_{2}+a_{2}\right) \otimes \mathbf{1}_{2}
\end{aligned}
$$

as before, and then proceed exactly as we did in the previous section. This leads to the $2+1$ dimensional U(2) Yang-Mills Lagrangian (31). However, it should be possible to make distinction between two singly wound (around $T^{2}$ ) membranes and a single membrane winding twice around one of the cycles of $T^{2}$. This distinction can be made as follows. We can add constant terms to (39) and define

$$
\begin{aligned}
& U_{1} \leftrightarrow\left(i \partial_{1}+a_{1}\right) \otimes \mathbf{1}_{2}+\left\langle A_{1}\right\rangle \\
& U_{2} \leftrightarrow\left(i \partial_{2}+a_{2}\right) \otimes \mathbf{1}_{2}+\left\langle A_{2}\right\rangle
\end{aligned}
$$


The constant terms $\left\langle A_{r}\right\rangle, r=1,2$ are $\mathrm{U}(2)$ Lie algebra valued. This addition does not affect the total 2-brane charge $Z_{12}$, since the commutator $\left[\left\langle A_{1}\right\rangle,\left\langle A_{2}\right\rangle\right]$ is traceless under $\operatorname{Tr}_{2}$. However, in order to leave the 2-brane charge density unaffected, we must require that the $\left\langle A_{1}\right\rangle$ and $\left\langle A_{2}\right\rangle$ commute.

Recall that the fluctuations $A_{1}, A_{2}$ around the background are defined by

$$
\begin{aligned}
& X_{1}=U_{1}+A_{1} \leftrightarrow\left(i \partial_{1}+a_{1}\right) \otimes \mathbf{1}_{2}+\left\langle A_{1}\right\rangle+A_{1} \\
& X_{2}=U_{2}+A_{2} \quad \leftrightarrow \quad\left(i \partial_{2}+a_{2}\right) \otimes \mathbf{1}_{2}+\left\langle A_{2}\right\rangle+A_{2} .
\end{aligned}
$$

From these relations it is apparent that the constant terms $\left\langle A_{r}\right\rangle$ can be interpreted as constant backround values of the gauge fields $A_{r}$ in the $\mathrm{U}(2)$ Yang-Mills Lagrangian (31). Thus, they can be used [7] to induce gauge holonomies $\mathcal{U}_{r}$, through the Wilson lines

$$
\mathcal{U}_{r}=P \exp \left\{i \oint_{r} A\right\}
$$

around the cycles of $T^{2}$. When the background values are both zero, the holonomies are trivial,

$$
\mathcal{U}_{1}=\mathcal{U}_{2}=\mathbf{1}_{2}
$$

around both cycles. From the IIA point of view, we then have a bound state of two D2-branes (and D0-branes), both winding once around the two cycles of $T^{2}$. But if we set

$$
\begin{aligned}
\left\langle A_{1}\right\rangle & =\frac{\pi}{2 L_{1}}\left(\begin{array}{cc}
-1 & 1 \\
1 & -1
\end{array}\right) \\
\left\langle A_{2}\right\rangle & =0
\end{aligned}
$$

we get a non-trivial holonomy

$$
\mathcal{U}_{1}=\left(\begin{array}{ll}
0 & 1 \\
1 & 0
\end{array}\right)
$$

In this case, the result is interpreted as a single D2-brane (with D0-branes) which winds twice around the $X_{1}$ cycle of $T^{2}$. Both cases yield the same 2-brane charge $Z_{12}$ which is twice the charge (38) of a single membrane. Generalizations to other winding membranes can be obtained in similar manner.

\subsection{Longitudinal fivebrane backgrounds $(p=4)$}

The fivebrane of M theory admits a simple description in Matrix theory only if it is wrapped around the longitudinal direction. Since the fivebrane is boost invariant in its world volume directions, going to the infinite momentum frame is more involved than for 
the transverse membrane. Momentum is instead added by superimposing a gravitational wave solution. However, in the case of a single fivebrane such a configurations necessarily includes nonzero membrane charge as well. To obtain a configuration with vanishing membrane charge one can take two fivebrane solutions wit mutually opposite membrane charges and then combine them. In this section we will examine fluctuations around both types of backgrounds. First, let us summarize the backrounds and their properties:

(i) an infinite longitudinal fivebrane with transverse membrane charge density; this reduces in $\mathrm{D}=10$ to a type IIA non-marginal bound state $4+2 \perp 2+0$

(ii) infinite longitudinal fivebranes with transverse leftmoving oscillations carrying momentum density in 11 th direction; this reduces in $\mathrm{D}=10$ to a marginally bound $4 \| 0$ configuration

In case (i), in the $N \rightarrow \infty$ limit the background is represented by

$$
U_{r} \leftrightarrow i \partial+a_{r} \quad r=1,2,3,4
$$

where $a_{r}$ is a $\mathrm{U}(1)$ background field with a field strength $f_{r s}$

$$
\left[U_{r}, U_{s}\right]=i f_{r s}
$$

with two nonzero constant components $f_{12}, f_{34}$ describing magnetic flux (2-brane charge) densities in 1-2 and 3-4 planes. A similar analysis as in Section 2.1 yields an effective Lagrangian for the bosonic fluctuations,

$$
\mathcal{L}_{B}=\sigma_{0} \int d^{4} x \frac{1}{2 g}\left\{-\frac{1}{2}\left(F_{\mu \nu}\right)^{2}+\left(\partial_{\mu} \phi_{i}\right)^{2}-f_{r s} F_{r s}-\frac{1}{2}\left(f_{r s}\right)^{2}\right\}
$$

where $\sigma_{0}$ is the D0-brane density. The relation between $\sigma_{0}$ and the longitudinal fivebrane charge density is discussed in the next example.

In case (ii), the background is represented by covariant derivatives with a selfdual U(2) background field:

$$
U_{r} \leftrightarrow i \partial_{r} \mathbf{1}_{2}+a_{r} \quad r=1,2,3,4
$$

where

$$
\begin{array}{ll}
a_{1}=0 & a_{3}=0 \\
a_{2}=c x_{2} \sigma^{3} & a_{4}=c x_{4} \sigma^{3} .
\end{array} .
$$

The \pm 1 diagonal elements of $\sigma^{3}$ represent the superposition of two solutions carrying opposite membrane charge. Note that the background breaks the $\mathrm{U}(2)$ symmetry to $\mathrm{U}(1)^{2}$. The field strength satisfies the selfduality condition

$$
f_{p q}=\tilde{f}_{p q}=\frac{1}{2} \epsilon_{p q r s} f_{r s} .
$$

\footnotetext{
${ }^{3}$ For earlier studies of such background configurations in non-abelian gauge theories, see e.g. 12 .
} 
This configuration carries a longitudinal 5-brane charge density

$$
\begin{aligned}
2 \cdot z_{1234} & =\frac{1}{8 \pi^{2}} \epsilon_{p q r s} \operatorname{Tr}\left[U_{p} U_{q} U_{r} U_{s}\right] \\
& =\frac{1}{16 \pi^{2}} \operatorname{Tr}\left[f_{p q} \tilde{f}_{p q}\right]=2 \cdot\left(\frac{c}{2 \pi}\right)^{2},
\end{aligned}
$$

where the prefactor 2 represents the presence of two fivebranes. The fluctuations $A_{0}, A_{r}, \phi_{i}$ about the background are represented by $\mathrm{U}(2)$ Lie algebra valued fields in $4+1$ dimensions:

$$
\begin{aligned}
A_{0} & \leftrightarrow A_{0}^{0}(x) \mathbf{1}_{2}+A_{0}^{a}(x) T^{a} \\
A_{r} & \leftrightarrow A_{r}^{0}(x) \mathbf{1}_{2}+A_{r}^{a}(x) T^{a} \\
\phi_{i} & \leftrightarrow \phi_{i}^{0}(x) \mathbf{1}_{2}+\phi_{i}^{a}(x) T^{a} .
\end{aligned}
$$

where $T^{a}$ are $\mathrm{SU}(2)$ generators. The effective action for the fluctuations is found to be

$$
\mathcal{L}_{B}=\sigma_{0} \int d^{4} x \frac{1}{2 g} \operatorname{Tr}_{2}\left\{-\frac{1}{2}\left(F_{\mu \nu}\right)^{2}+\left(D_{\mu} \phi_{i}\right)^{2}+\frac{1}{2}\left[\phi_{i}, \phi_{j}\right]^{2}-f_{r s} F_{r s}-\frac{1}{2}\left(f_{r s}\right)^{2}\right\} .
$$

For a transverse membrane, the D0-brane density $\sigma_{0}$ was inversely related (15) to the membrane charge density. Similarly, for longitudinal fivebranes $\sigma_{0}$ is inversely related to the longitudinal fivebrane charge density,

$$
\sigma_{0}=\frac{1}{(2 \pi)^{4} z_{1234}} .
$$

The energy density of the configuration is the sum of fivebrane plus gravitational wave contributions $H=T+p_{11}$. The infinite momentum frame Hamiltonian is found by subtracting $p_{11}: H_{\mathrm{IMF}}=T$. T is found from the constant term in (47),

$$
T=2 T_{4}=2 \frac{1}{(2 \pi)^{4}} T_{0} .
$$

This is as expected, since two longitudinal fivebranes are interpreted as two D4-branes in the IIA theory.

\section{$3 \quad$ Scattering of branes}

Recently, there have been several studies of brane-brane scattering in the context of Matrix theory [6, 14, 15, 16, 17, 18, 19, 20, 21]. These calculations have demonstrated agreement between 11 dimensional supergravity and Matrix theory at one loop and even

\footnotetext{
${ }^{4}$ Subtler issues and possible discrepancies have also been discussed, see [22, 23].
} 
two loops [19]. For Matrix theory compactified on torii, such calculations amount to loop diagrams in Super-Yang-Mills theory on the dual torus with magnetic backround fields encoding the brane configuration and Higgs field VEVs specifying the kinematics of the scattering problem. From the discussion in Section 2, it should be obvious that a similar correspondence applies also for scattering in uncompactified M(atrix) theory involving infinite branes.

Note that all the effective actions (19, 31, 43, 47) can be rewritten in a form where the magnetic background fields are combined with the fluctuation fields. Introducing a $\mathrm{U}(\mathrm{k})$ gauge field $\mathcal{A}_{\mu}$,

$$
\begin{aligned}
& \mathcal{A}_{0}=A_{0} \\
& \mathcal{A}_{r}=a_{r}+A_{r}
\end{aligned}
$$

and denoting its field strength by $\mathcal{F}_{\mu \nu}$, the actions (19, 31, 43, 47) can be written in the form

$$
\mathcal{L}_{B}=\frac{1}{2 g} \sigma_{0} \int d^{p} x \operatorname{Tr}_{k}\left\{-\frac{1}{2}\left(\mathcal{F}_{\mu \nu}\right)^{2}+\left(\mathcal{D}_{\mu} \phi_{i}\right)^{2}+\frac{1}{2}\left[\phi_{i}, \phi_{j}\right]^{2}\right\}
$$

where the covariant derivative $\mathcal{D}_{\mu}$ is taken with respect to the field (50) which includes the background. Now, after including fermions, gauge fixing and ghost terms, we can compute scattering amplitudes from loop diagrams in the appropriate background fields which encode the information about the scattering objects and kinematical setup.

\subsection{D0 - D6+D0 Scattering}

We will perform a scattering calculation involving configurations of D6-branes and D0branes. Among various other configurations, G. Lifschytz studied the scattering of $6+4+2+0$ bound states from D0-branes [15]. (Further discussion can be found in [22].) Recently, W. Taylor [4 showed how to compose configurations of D6 branes and D0-branes which do not carry D4 or D2 brane charges. Such backgrounds can be denoted by

$$
U_{r}=i \partial_{r} \mathbf{1}_{4}+a_{r} \quad r=1,2, \ldots 6
$$

with a background $\mathrm{U}(4)$ gauge field

$$
\begin{array}{lll}
a_{1}=0 & a_{3}=0 & a_{5}=0 \\
a_{2}=c x_{2} \mu_{1} & a_{4}=c x_{4} \mu_{2} & a_{6}=c x_{6} \mu_{3}
\end{array}
$$

\footnotetext{
${ }^{5}$ These configurations are classically stable up to quadratic order. They are thought to be related to the supergravity black hole solutions of [24] carrying 0-brane and 6-brane charges.
} 
with traceless U(4) matrices

$$
\begin{aligned}
& \mu_{1}=\operatorname{diag}(1,1,-1,-1) \\
& \mu_{2}=\operatorname{diag}(1,-1,-1,1) \\
& \mu_{3}=\operatorname{diag}(1,-1,1,-1) .
\end{aligned}
$$

Analogously to (45), the above configuration gives a charge density

$$
\frac{i}{48 \pi^{3}} \epsilon_{p q r s t u} \operatorname{Tr}_{4}\left(U_{p} U_{q} U_{r} U_{s} U_{t} U_{u}\right)=4 \cdot\left(\frac{c}{2 \pi}\right)^{3} .
$$

We have examined the scattering of D0 particles from Taylor's D6+D0 brane configurations and calculated the potential between a these objects. The D6+D0 configuration can be understood in the same way as the fivebrane with vanishing membrane charge of Section 2.3. It represents a superposition of four $6+4+2+0$ solutions which are chosen to give vanishing D4-brane and D2-brane charges when combined. The scattering calculation that follows reveals this structure, in as much as the potential is found to be four times that between a D0 particle and a $6+4+2+0$ state. In the Matrix theory 1-loop calculation (which is the same as the 1-loop calculation in the effective theory (50), as we argued above), we encounter the following determinants (the boson, ghost and fermion contributions in the background gauge)

$$
\begin{aligned}
& \operatorname{det}^{-3}\left\{\left(-\partial_{\tau}^{2}+H+2 c\right) \mathbf{1}_{4}\right\} \operatorname{det}^{-3}\left\{\left(-\partial_{\tau}^{2}+H-2 c\right) \mathbf{1}_{4}\right\} \\
& \operatorname{det}^{-2}\left\{\left(-\partial_{\tau}^{2}+H\right) \mathbf{1}_{4}\right\} \\
& \operatorname{det}\left\{\left(-\partial_{\tau}^{2}+H+3 c\right) \mathbf{1}_{4}\right\} \operatorname{det}^{3}\left\{\left(-\partial_{\tau}^{2}+H+c\right) \mathbf{1}_{4}\right\} \\
& \operatorname{det}^{3}\left\{\left(-\partial_{\tau}^{2}+H-c\right) \mathbf{1}_{4}\right\} \operatorname{det}\left\{\left(-\partial_{\tau}^{2}+H-3 c\right) \mathbf{1}_{4}\right\},
\end{aligned}
$$

where

$$
H=r^{2}+c\left(2\left(n_{1}+n_{2}+n_{3}\right)+3\right) .
$$

Here $r$ is the distance between the D0-brane and the $6+0$ configuration and $n_{1}, n_{2}, n_{3}=$ $0,1, \ldots$. These determinants are the same as those in the calculation by Lifschytz of the D0 $-6+4+2+0$ potential, except for additional $4 \times 4$ unit matrix factors. The unit matrices signal that the D0-brane sees the four $6+4+2+0$ sublayers in the $6+0$ configuration, thus the potential between the $6+0$ configuration and the D0-brane will be four times that obtained by Lifschytz [15]:

$$
V(r)=4 \cdot \frac{3}{16} \frac{c}{r}
$$

The corresponding supergravity result is easily obtained by considering the $6+0$ configuration as a probe moving in the D0-brane background. The calculation is performed in the same manner as by Chepelev and Tseytlin [20], who considered various configurations 
involving 4-branes and 5-branes. Their paper also collects a number of useful formulae which we will draw from in the following. The effective action for the $6+0$ configuration is given by|

$$
S_{6}=\int d^{7} \xi \operatorname{Tr}\left\{-T_{6} e^{-\phi} \sqrt{-\operatorname{det}\left[g_{\mu \nu} \frac{\partial X^{\mu}}{\partial \xi^{i}} \frac{\partial X^{\nu}}{\partial \xi^{j}}+F_{i j}\right]}+\mu_{6}\left(C_{0} F_{12} F_{34} F_{56}+\cdots\right)\right\},
$$

where $\cdots$ indicates various Chern-Simons terms which are irrelevant for our present purposes. In our units, we have $\mu_{6}=T_{6}$. The field strength in the worldvolume is given by $F_{12}=F \mu_{1}, F_{34}=F \mu_{2}, F_{56}=F \mu_{3}$, with $\mu_{1,2,3}$ defined in (53). The spacetime fields to be inserted into $S_{6}$ are those induced by a D0-brane source "smeared" in directions $x^{1}, \ldots, x^{6}$ :

$$
\begin{aligned}
d s^{2} & =-H_{0}^{-1 / 2} d t^{2}+H_{0}^{1 / 2}\left(d x_{1}^{2}+\cdots+d x_{9}^{2}\right) \\
e^{-\phi} & =H_{0}^{-3 / 4} ; \quad C_{0}=H_{0}^{-1}-1 \\
H_{0} & =1+\frac{Q_{0}^{(6)}}{r} ; r^{2}=x_{7}^{2}+x_{8}^{2}+x_{9}^{2} \\
Q_{0}^{(6)} & =\frac{g}{2}(2 \pi)^{5 / 2} .
\end{aligned}
$$

Substituting into $S_{6}$ yields

$$
S_{6}=-4 T_{6} \int d^{7} x\left\{H_{0}^{-1}\left(H_{0}+F^{2}\right)^{3 / 2}-\left(H_{0}^{-1}-1\right) F^{3}\right\} .
$$

To compare with the Matrix theory result we should take $r, F \rightarrow \infty$, corresponding to the large distance interaction between objects in the infinite momentum frame of $\mathrm{M}$ theory. Then

$$
S_{6} \approx-4 T_{6} \int d^{7} x\left\{\frac{3}{8} \frac{H_{0}}{F}+F^{3}+\frac{3}{2} F\right\} .
$$

Now we can read off the potential,

$$
V(r) \approx 4 \cdot \frac{3}{8} \frac{T_{6} Q^{(6)}}{F r}=4 \cdot \frac{3}{16} \frac{1}{F r},
$$

in agreement with the Matrix theory result (55), upon recalling the inverse relation between the magnetic fields $c=1 / F$.

\section{Conclusions}

We have seen that by taking the $N \rightarrow \infty$ limit of Matrix theory in a particular way, it is possible to recover much of the known physics of D-branes in IIA theory. One aspect

\footnotetext{
${ }^{6}$ For the effective action we will use Tseytlin's truncated version of the non-abelian Born-Infeld action, valid in cases, such as the present one, where the worldvolume field strengths commute.
} 
which proved to be problematic, as discussed in Section 2.1, was the relation between the magnetic fields introduced in the Matrix and IIA descriptions. We saw that the effective action for a D-brane came out incorrectly due to the background magnetic field being the inverse of what was needed for agreement with the Born-Infeld action. This disagreement is especially puzzling in light of a recent calculation of Polchinski and Pouliot 21] involving longitudinal momentum transfer between membranes. (Related discussion can be found in 25, 26].) The starting point for their calculation was the assumption that Matrix theory would yield the effective action for a D2-brane, including the correct magnetic field dependence. It would be interesting to see how this assumption can be reconsiled with our findings.

Finally, although in this paper attention has been restricted to the $N \rightarrow \infty$ limit, it would be useful to investigate D-branes at finite N. Despite the absense of infinite BPS branes for such $\mathrm{N}$, it should be possible to compare various processes with the predictions of supergravity compactified along a null direction. Agreement would provide substantial evidence for the conjecture of [5].

\section{Acknowledgements}

We would especially like to thank Soo-Jong Rey for discussions which motivated this investigation.

\section{References}

[1] T. Banks, W. Fischler, S.H. Shenker, L. Susskind, M theory as a matrix model: A Conjecture, Phys. Rev. D55, 5112 (1997), (hep-th/9610043).

[2] T. Banks, N. Seiberg, and S. Shenker, Branes from matrices, Nucl. Phys. B490, 91 (1997), (hep-th/9612157).

[3] S.-J. Rey, private communication.

[4] W. Taylor, IV, Adhering 0-branes to 6-branes and 8-branes, hep-th/9705116.

[5] L. Susskind, Another conjecture about M(atrix) theory, hep-th/9704080.

[6] O. Aharony and M. Berkooz, Membrane dynamics in M(atrix) theory, Nucl. Phys. B491, 184 (1997) (hep-th/9611215).

[7] A. Hashimoto, Perturbative dynamics of fractional strings on multiply wound D strings, hep-th/9610250; J. Polchinski, TASI lectures on D-branes, hep-th/9611050. 
[8] A. Hashimoto and W. Taylor, IV, Fluctuation spectra of tilted and intersecting D-branes from the Born-Infeld action, hep-th/9703217.

[9] W. Taylor, IV, D-brane field theory on compact spaces, Phys.Lett. B394, 283 (1997), (hep-th/9611042); O.J. Ganor, S. Ramgoolam, W. Taylor, IV, Branes, fluxes and duality in M(atrix) theory, hep-th/9611202.

[10] B. de Wit, K. Peeters and J.C. Plefka, Supermembranes with winding, hepth/9705225.

[11] L. Susskind, T duality in M(atrix) theory and $S$ duality in field theory, hepth/9611164.

[12] H. Leutwyler, Constant gauge fields and their quantum fluctuations, Nucl. Phys. B179, 129 (1981); P. van Baal, SU(N) Yang-Mills solutions with constant field strength on $T^{4}$, Commun. Math. Phys. 94, 397 (1984); Some results for $S U(N)$ gauge fields on the hypertorus, Commun. Math. Phys. 85, 529 (1982).

[13] G. Lifschytz, Probing bound states of D branes, hep-th/9610125.

[14] G. Lifschytz and S.D. Mathur, Supersymmetry and membrane interactions in $M$ (atrix) theory, hep-th/9612087.

[15] G. Lifschytz, Four brane and six brane interactions in M(atrix) theory, hepth/9612223.

[16] D. Berenstein and R. Corrado, M(atrix) theory in various dimensions, hepth/9702108.

[17] G. Lifschytz, A note on the transverse five-brane in M(atrix) theory, hepth/9703201.

[18] J.M. Pierre, Interactions of eight-branes in string theory and M(atrix) theory, hep-th/9705110.

[19] K. Becker and M. Becker, A Two loop test of M(atrix) theory, hepth/9705091.

[20] I. Chepelev and A.A. Tseytlin, Long distance interactions of D-brane bound states and longitudinal 5-brane in M(atrix) theory, hep-th/9704127. 
[21] J. Polchinski and P. Pouliot, Membrane scattering with $M$ momentum transfer, hep-th/9704029.

[22] V. Balasubramanian and F. Larsen, Relativistic brane scattering, hepth/9703039.

[23] O.J. Ganor, R. Gopakumar and S. Ramgoolam, Higher loop effects in M(atrix) orbifolds, hep-th/9705188.

[24] H. Sheinblatt, Statistical entropy of an extremal black hole with 0-brane and 6-brane charge, hep-th/9705054.

[25] N. Dorey, V.V. Khoze, and M.P. Mattis, Multi-instantons, threedimensional gauge theory, and the Gauss-Bonnet-Chern theorem, hepth/9704197.

[26] T. Banks, W. Fischler, N. Seiberg and L. Susskind, Instantons, scale invariance, and Lorentz invariance in Matrix theory, hep-th/9705190. 